\title{
Aux origines de l'Église arménienne catholique de Jérusalem
}

\section{Sossie Andézian}

\section{(2) OpenEdition \\ 1 Journals}

Édition électronique

URL : http://journals.openedition.org/assr/22321

DOI : 10.4000/assr.22321

ISSN : $1777-5825$

Éditeur

Éditions de l'EHESS

\section{Édition imprimée}

Date de publication : 1 septembre 2010

Pagination : 47-69

ISBN : 978-2-7132-2255-9

ISSN : 0335-5985

\section{Référence électronique}

Sossie Andézian, "Aux origines de l'Église arménienne catholique de Jérusalem », Archives de sciences sociales des religions [En ligne], 151 | juillet-septembre 2010, mis en ligne le 20 octobre 2010, consulté le 19 avril 2019. URL : http://journals.openedition.org/assr/22321 ; DOI : 10.4000/assr.22321 


\section{Sossie Andézian}

\section{Aux origines de l'Église arménienne catholique de Jérusalem}

Le 17 septembre 2005, le catholicos-patriarche arménien catholique, BedrosNersès XIX Tarmouni ${ }^{1}$ se rend de son siège de Beyrouth à Jérusalem pour $y$ commémorer le centenaire de l'église Notre-Dame des Douleurs. Il inaugure également la crypte restaurée à l'occasion du millenium. Bâtie sur le site de la IV $^{\mathrm{e}}$ station du Chemin de croix, l'église s'élève au centre d'un couvent composé d'une petite chapelle, site de la III $^{\mathrm{e}}$ station, d'un presbytère, d'un hôtel pour pèlerins et de deux immeubles d'habitation. Propriété du patriarcat arménien catholique, d'une superficie de $1770 \mathrm{~m}^{2}$, le complexe est situé dans le quartier musulman de la vieille ville, à l'angle de la Via Dolorosa et de la rue al-Wad. Face à l'hospice autrichien au nord, il est séparé par un établissement juif au sud et par deux zāwiya-s des ordres Qadiriyya et Naqshabandiyya à l'est. Portant le titre $d^{\prime}$ " exarchat ${ }^{2}$ du patriarcat arménien catholique ", l'ensemble fonctionne à la fois comme lieu de pèlerinage pour les catholiques et comme paroisse pour ceux de rite arménien, dont une dizaine de familles occupent les logements contre des loyers modiques.

Construire un lieu de pèlerinage et d'accueil pour les nationaux de Constantinople et d'ailleurs, et pour les catholiques du monde entier, sont les deux objectifs de cette Église qui entreprend son installation à Jérusalem en 1854. Progressivement se fait jour la volonté de développer le rite dans la ville, par la conversion des Arméniens apostoliques d'une part, " frères schismatiques restés dans l'erreur ", celle des Arméniens passés au rite latin ou aux rites protestants d'autre part. L'action s'inscrit dans le mouvement d'expansion du catholicisme en Terre sainte et sa réappropriation des lieux saints et des sanctuaires "ensevelis » sous des bâtiments musulmans.

1. Catholicos: titre des chefs de l'Église arménienne $(c f$. infra). Le prénom Bedros, Pierre en français, fait référence à l'apôtre dont les catholicos arméniens catholiques se disent les successeurs.

2. Dénomination du nouveau code oriental des vicariats ; ici j'emploierai le terme vicariat, en usage à l'époque. 


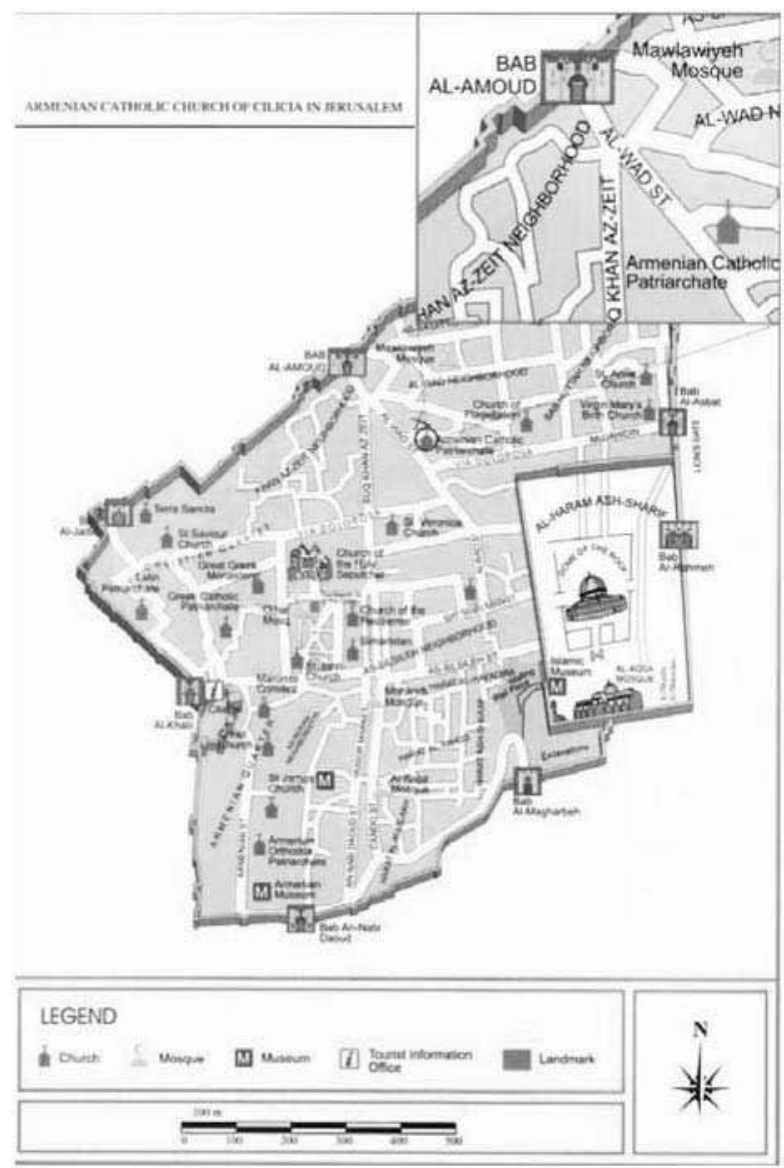

Plan de la vieille ville de Jérusalem (Source : Brochure produite à l'occasion de l'inauguration de la crypte en 2005. Center for advanced technology and design)

Les moyens mis en œuvre seront différents selon les périodes et selon les vicaires. Une constante demeure : le caractère de lutte permanente de l'opération, contre les pouvoirs politiques locaux et étrangers, contre l’Église arménienne apostolique, contre les autres Églises chrétiennes, contre les autres Églises catholiques orientales et contre certains éléments de l'Église latine, voire contre des éléments internes. Les deux projets, établissement d'un lieu de pèlerinage international et d'une paroisse arménienne catholique, se réaliseront malgré tout. Cependant, il s'agira d'une petite communauté, atteignant le nombre d'un millier d'individus environ à son apogée ${ }^{3}$, qui sera constituée non pas tant par des convertis que

3. Chiffre incluant les membres de la paroisse de Jordanie qui fait partie du diocèse de Jérusalem. Selon le recensement britannique de 1931, le nombre des Arméniens catholiques était de trois cent trente (E. Mills, 1931). Les chiffres varient selon qu'on inclut les Arméniens 
par des réfugiés rescapés du génocide arrivés en Palestine dans les années vingt. L'Église arménienne catholique sera officiellement reconnue à Jérusalem avec le titre de "vicariat patriarcal » et la communauté arménienne catholique une des communautés confessionnelles avec son statut personnel spécifique. Le lieu de pèlerinage international se développera fortement au fil du temps. À partir de 1948 , la population se réduira sensiblement. La paroisse réussira toutefois à maintenir ses activités. Mais, progressivement, le caractère international s'accentuera, pour aboutir à la fondation, dans la crypte, d'un lieu d'adoration perpétuelle du saint sacrement et sa dédicace comme "Centre de la Paix à Jérusalem » par le nonce apostolique, le 25 mars 2009, jour de l'Annonciation ${ }^{4}$.

C'est le processus de fondation de ce lieu dans le contexte particulier de la Terre sainte de la seconde moitié du XIX ${ }^{\mathrm{e}}$ siècle, son inscription dans l'espace de Jérusalem et son intégration dans l'histoire du catholicisme oriental d'une part, dans l'histoire du peuple arménien et de son Église d'autre part, qui est présenté ici. On en suivra les différentes phases de fondation et re-fondation, à la fois matérielle et symbolique, ainsi que l'évolution des représentations religieuses qui lui donneront corps. On verra notamment quels usages sont faits des vestiges chrétiens de la ville, des traditions écrites et orales, pour inventer ou réinventer un lieu saint dans un espace saturé de sacré. On assistera à l'émergence d'une nouvelle communauté confessionnelle qui prendra place dans la nouvelle configuration de la population chrétienne de Jérusalem.

\section{Naissance d'une communauté confessionnelle}

L'Église arménienne est dite apostolique en référence aux apôtres Thaddée et Barthélémy, "les évangélisateurs de l'Arménie ", ou grégorienne en référence à son fondateur l'évêque saint Grégoire l'Illuminateur. C'est une Église nationale, l'Arménie ayant adopté le christianisme comme religion d'État en $301^{5}$. Son chef, qui porte le titre de "Catholicos de tous les Arméniens ", est considéré comme le successeur de saint Grégoire. Le siège (catholicossat) est fixé à Etchmiadzine, à une vingtaine de kilomètres de la capitale Erevan. Sa position s'est renforcée depuis l'accession de l'Arménie à l'indépendance. Un second catholicossat, créé dans le royaume de Cilicie à Sis, au XIII , se trouve aujourd'hui au Liban où il avait été transféré après le génocide. Les patriarcats de Jérusalem et de Constantinople, fondés respectivement en 1311 et $1461^{6}$, sont toujours en activité.

catholiques de Jordanie ou pas, ce qui n'est pas toujours précisé dans les statistiques. Par ailleurs, la naturalisation des réfugiés arméniens se fait au cours des années trente; la plupart d'entre eux, encore en situation irrégulière à l'époque, ne sont pas recensés. On évaluait à quelque sept mille le nombre de réfugiés arméniens arrivés en Palestine au début du XXe siècle.

4. Zenit, l'organe du Vatican en ligne, http://www.zenit.org/article

5. Date officielle choisie par l'historiographie de l'Église mais contestée par les historiens qui situent l'événement une douzaine d'années plus tard.

6. Là encore, il s'agit de dates officielles. 
La christianisation du pays est favorisée par l'invention, au début du ve siècle, de l'alphabet arménien par le moine Mesrop Machtots dans le but de traduire les livres saints et d'évangéliser la population dans sa propre langue, alors que le pays est déchiré entre les empires perse et byzantin qui se partagent le territoire. La liturgie de langue arménienne remplace les textes byzantins et syriaques.

C'est une Église autocéphale suite à son rejet des décisions de Chalcédoine. Elle s'en tient aux trois premiers conciles, proclame l'union dans la personne du Christ des deux natures, divine et humaine, et reconnaît Marie Théotokos (Mère de Dieu). Et elle conteste l'appellation monophysite puisqu'elle avait anathémisé la doctrine d'Eutychès. Elle ne rejette pas le dogme de l'Incarnation mais plutôt la soumission à l'Empereur byzantin que l'adhésion à Chalcédoine entraînait (Ormanian, 2000).

L'Église arménienne catholique prend officiellement naissance en $1742^{7}$, avec la reconnaissance par le pape Benoît XIV de son chef, l'évêque Abraham Ardzivian qui avait demandé son rattachement à Rome. Exclu de l'Église arménienne apostolique et exilé, Ardzivian finira par trouver refuge dans la montagne libanaise, au Kesrouan, où le patriarche maronite lui réserve un très bon accueil. Il s'installe au couvent des moines antonins arméniens et prend le titre de "Catholicos de Cilicie pour les Arméniens catholiques ». Son successeur fonde, à proximité, le monastère de Bzommar, devenu aujourd'hui séminaire et résidence d'été (Djourian, 1990).

En 1830, la communauté arménienne catholique de Constantinople, jusquelà incluse dans le millet ${ }^{8}$ arménien sous la juridiction du patriarche arménien apostolique, obtient un statut de millet autonome. Si la création de ce millet met fin aux persécutions subies par les Arméniens de ce rite et leur accorde la liberté de culte, elle entraîne de nouveaux problèmes, avec la hiérarchie de l'Église catholique d'une part, entre clercs et laïcs d'autre part. La communauté n'est pas soumise à l'autorité spirituelle du catholicos-patriarche du Liban mais à celle d'un archevêque-primat de Constantinople nommé par le pape (alors que le premier est élu, selon la tradition de l'Église arménienne qui accorde une grande place aux laïcs ${ }^{9}$, par un synode de clercs et de laïcs puis confirmé par Rome).

7. La fondation de cette Église était préparée depuis longtemps, des Arméniens ayant été convertis au catholicisme par les missionnaires latins à partir du XVI e siècle. Ardzivian lui-même s'était converti dans sa jeunesse. Certains représentants de l'Église soutiennent qu'un courant chalcédonien est resté en union avec Rome. Des catholicos arméniens entretiennent des liens plus ou moins forts avec Rome selon les époques, les croisades ayant constitué un moment privilégié. L'abbé Mekhitar de Sébaste crée la congrégation des Mékhitaristes, en 1701, dont le rôle dans le développement et la diffusion de la langue et de la culture arméniennes est bien connu (Whooley, 2004).

8. Millet : système communautaire établi dans l'Empire ottoman, et réformé au XIXe siècle, regroupant les non musulmans au sein de structures fondées sur l'appartenance confessionnelle qui bénéficiaient de l'autonomie administrative dans la gestion de la vie religieuse et culturelle ainsi que celle du statut personnel de leurs membres.

9. La Constitution de la communauté arménienne apostolique, ratifiée en 1863, conférait aux laïcs de plus grandes prérogatives au sein de l'Église. La Constitution de 1888 rétablira ces prérogatives chez les catholiques. 
Un chef civil, patrik, représente le millet auprès de la Sublime Porte et en administre les biens. Pour mettre fin aux tensions résultant du dédoublement de l'autorité spirituelle, à la mort du titulaire du siège du Liban, en 1866, le nouveau candidat, Antoine Hassoun, qui était archevêque-primat de Constantinople et soutenu par Rome, réunit les deux fonctions. Avec l'aide de la France, il obtient également le titre de chef civil, et le siège patriarcal est transféré de Bzommar à Constantinople où il restera jusqu'au génocide, avant de revenir au Liban en 1928.

Deux autres initiatives de Rome, soutenues par Hassoun, suscitent la colère non seulement des laïcs, mais également d'une partie du clergé, provoquant un nouveau schisme et installant l'Église dans une crise longue de plusieurs années : la bulle Reversurus, promulguée par Pie IX en 1867, qui exclut l'Assemblée des laïcs des instances de nomination des prélats, et la déclaration du Concile de 1869-1870 de la primauté et de l'infaillibilité du pape. L'élection de Léon XIII, en 1878, rétablit l'ordre, Rome s'abstenant désormais de s'immiscer dans les affaires internes de l'Église (Whooley, 2004).

La fondation de l'Église arménienne catholique de Jérusalem se fait dans ce contexte de crise, qui aura des répercussions certaines sur la réalisation du projet.

\section{La montée à Jérusalem}

La présence arménienne à Jérusalem, qui remonte aux premiers siècles chrétiens, est généralement associée au patriarcat arménien apostolique administré par la confrérie Saint-Jacques, ainsi qu'aux lieux saints dont il se partage la propriété et/ou le contrôle avec les Églises grecque orthodoxe et latine. Situé sur le Mont Sion, le siège du patriarcat, construit autour de la cathédrale des SaintsJacques (le Majeur et le Mineur), est un vaste complexe entouré de murailles et composé de plusieurs chapelles, d'un monastère, d'un parc résidentiel, d'une école proposant un cycle complet d'enseignement, d'une bibliothèque de manuscrits et d'une bibliothèque générale, d'un musée et d'une librairie. Un séminaire, une imprimerie ainsi qu'un cimetière constituent deux autres îlots de l'autre côté de la rue. Avec les maisons, les immeubles et les magasins alentour, l'ensemble forme le quartier arménien de Jérusalem, un des quatre quartiers de la vieille ville qui occupe le sixième de sa superficie globale.

Le projet de fondation d'une Église arménienne catholique à Jérusalem au cours de la seconde moitié du XIX ${ }^{\mathrm{e}}$ siècle peut être lu comme une tentative de positionnement sur la scène internationale qu'est devenue la ville sainte. La chute d'Acre, devant l'Égyptien Ibrahim Pacha, ainsi que les ingérences occidentales avaient transformé Jérusalem en véritable capitale provinciale sous l'autorité directe du sultan. L'affaiblissement de l'Empire ottoman va conduire les puissances occidentales à se poser en protectrices des chrétiens, qui feront l'objet de luttes d'influence entre elles. Leurs intérêts ne sont pas que religieux mais surtout 
stratégiques, politiques et économiques. Les consulats se multiplient, chaque pays essayant d'attirer ses missionnaires et ses ressortissants et de développer établissements religieux, culturels, caritatifs et économiques. Le protectorat des Arméniens catholiques sera assuré, comme celui de tous les catholiques, par la France, au moins jusqu'à la défaite de 1870 devant la Prusse, où elle sera de plus en plus concurrencée par l'Italie avec les franciscains mais également par le Vatican qui tient à consolider sa souveraineté étatique ${ }^{10}$. Aussi les difficultés auxquelles se heurte le projet relèvent-elles autant de la sphère religieuse que des sphères administrative, politique et diplomatique. Elles se présentent à chaque étape d'un processus long d'un demi-siècle : achat du terrain - attestation, réinvention et appropriation d'un lieu saint - établissement d'un centre de pèlerinage - édification de l'église - formation d'une communauté confessionnelle.

\section{Appropriation d'un site Via Dolorosa}

Selon les archives du vicariat ${ }^{11}$, le prêtre Sérovpé Tavitian y est envoyé, en 1854, par le catholicos Asdvadzadourian, avec la mission de trouver un terrain. Il séjourne au patriarcat latin, qui vient d'être restauré en 1847, et y travaille comme procureur et comme enseignant au séminaire (Duvignau, 1972). Il y reste jusqu'en 1865. Son choix se porte sur un site connu sous le nom de Hammam alSultan. Il achète les ruines du hammam, et achète ou loue pour cent ans plusieurs maisons délabrées ainsi que les terrains vagues environnants. L’opération est financée par un notable arménien de Constantinople, Andon Bey Missirlian. Le patriarcat acquiert le terrain en 1856 et la propriété est inscrite au registre foncier de Jérusalem. Mais de nombreuses interventions auprès de la Sublime Porte seront nécessaires pour contourner les obstacles administratifs.

Des archives du ministère français des Affaires étrangères révèlent le rôle de la France dans l'acquisition de ces terrains (Sroor, 2005). Celle-ci se fait à la faveur des réformes ou tanzimāt touchant au statut des biens waqf-s, qui deviennent propriétés de l'État. Ils sont mis à la vente ou donnés, comme dans le cas de l'église Sainte-Anne, attribuée par le Sultan Abd al-Majid à la France pour son aide dans la guerre de Crimée contre la Russie (Trimbur, 2000). L'article 27 du

10. Pour plus de développements, voir l'article très synthétique de C. Mouradian, 1999.

11. Non encore établies selon des normes internationales, ces archives, que je classe moimême depuis plusieurs années, regroupent des documents administratifs concernant le domaine, des correspondances avec diverses instances (autorités publiques, institutions ecclésiastiques, corps diplomatique), des correspondances privées, des registres paroissiaux, des rapports d'activités, des diaires, des autobiographies. N'ont été utilisés que les documents datés et signés. Plusieurs histoires de l'Église ont été ébauchées par différents vicaires mais sont restées inédites. Deux articles en arménien, parus dans la revue Pazmaveb (Sarkissian, 1906) des mékhitaristes de Venise et dans la revue Avédik (anonyme, 1945) du Patriarcat arménien catholique, ainsi qu'un article en anglais (Stephan, 1933), se fondent sur ces textes mais aussi sur des observations directes et des entretiens. Par ailleurs, la revue La Terre Sainte constitue une source inestimable pour l'histoire de la fondation du lieu. 
Hatti-Hamayoun $^{12}$, du 18 février 1856, autorise les étrangers à acquérir des biens immobiliers, à condition que ceux-ci se conforment aux lois du pays et aux règlements de la police locale (Sroor, op. cit.) C'est surtout à la suite de la promulgation du code de propriété des étrangers, en 1867, que les congrégations religieuses vont pouvoir le faire. Le patriarche latin, aidé par le consul de France, cherche à acheter des terrains dans ce quartier musulman autour du Haram alSharif, établi par la tradition chrétienne comme le chemin de la Passion : la tour d'Antonia, Ecce Homo, l'Arche de Pilate, les sites de la Flagellation, de la Condamnation et de la Chute du Christ sous la croix, celui de la Rencontre avec sa Mère... Pour les catholiques, il s'agit de reprendre possession des vestiges des lieux saints chrétiens (Trimbur, op. cit.) Mais c'est aussi le quartier des institutions publiques et des résidences des autorités politiques. C'est là que s'installent les consuls occidentaux.

Duvignau (op. cit.) souligne que le patriarche arménien catholique entreprend d'acquérir un lieu en Terre sainte à l'instigation du patriarche latin Joseph Valerga, qui avait constaté un mouvement de conversion d'Arméniens apostoliques au rite latin. C'est l'époque où le Vatican change de stratégie dans son action missionnaire et prône la conversion au catholicisme des Orientaux par des prêtres indigènes ${ }^{13}$. Dans un premier temps, le gouvernement ottoman refuse de signer l'acte de vente en invoquant la proximité de l'Esplanade des mosquées et le voisinage des zāwiya-s. Les consuls autrichien et britannique s'y opposent également (Sroor, op. cit.) Le premier, pourtant protecteur des catholiques, ne voit pas d'un bon œil l'installation d'un établissement chrétien concurrent en face de l'hospice autrichien en cours de construction. L'opposition du consul britannique, James Finn, relève de la guerre entre missions catholiques et missions protestantes qui se disputent les fidèles grecs orthodoxes et arméniens, et aussi entre puissances européennes pour leur rôle dans le contrôle des lieux saints (Mouradian, op. cit.) Dans une lettre adressée à son ministère des Affaires étrangères, suite à une enquête sur le site du hammam ${ }^{14}$, Finn juge inutile l'édification

12. Rescrit impérial du 18 février 1856, qui déclare l'égalité de tous les sujets de l'empire et qui garantit, en principe, aux communautés religieuses la liberté de culte et le droit d'administrer leurs biens.

13. «En partant de Rome, Mgr Valerga s'était vu remettre et instamment recommander l'Instruction publiée par la SC de la Propagande le 13 novembre 1845 sur l'obligation de promouvoir la création d'un clergé indigène dans les Missions " (Duvignau, 1972 : 102). Mais c'est le pape Léon XIII (1878-1903), qui manifestera un intérêt certain pour le christianisme oriental et prônera la "régénération de l'Orient par l'Orient ". Il favorisera la formation de clergés indigènes en mettant en place des séminaires orientaux pilotes, celui de Sainte-Anne à Jérusalem, en 1882, par exemple, séminaire grec-catholique confié aux Pères Blancs de Lavigerie (Trimbur, op.cit.) et le séminaire syrien-catholique, en 1903, dirigé par les pères bénédictins (Trimbur, 2002). Lavigerie était allé plus loin en appelant à la conversion des missionnaires occidentaux aux rites orientaux (Bouwen, 1992). L'année du jubilé épiscopal (1893-1894) constituera un tournant décisif avec l'organisation du Congrès eucharistique international à Jérusalem afin de témoigner de l'universalisme du catholicisme.

14. Archives of the Public Record Office, Londres (APROL, Fo 78/1217, 1856). 
d'une église arménienne catholique alors que Jérusalem ne compte que quatre familles de ce rite. Il confirme qu'il s'agit d'un bien du waqf Hassaki Sultane, fondé au XVI siècle au nom de Roxelane, l'épouse russe du sultan Suleiman; le hammam ainsi qu'un bâtiment adjacent ont été acquis par le consulat de France. Il souligne le caractère privilégié du site, à proximité du palais du gouvernement, où la France aurait acheté à une famille musulmane plusieurs biens immobiliers avec un seul contrat, afin de réduire les difficultés liées à la transformation des biens waqf-s. L'acte d'achat est passé au tribunal de Jérusalem le 25 novembre 1857. Finn reproche à la France d'avoir usé d'un stratagème pour acquérir ces terrains, destinés en réalité à la construction d'une église et d'un couvent par une communauté "qui n'était pas connue à Jérusalem et puis qui s'est appelée les Arméniens catholiques » (ibid.: 326).

\section{Procès de fondation}

Ce sera principalement l'œuvre du vicaire Hovaguim Toumayan qui remplace Tavitian en 1872. Outre la construction matérielle du lieu, il va en confirmer le statut de lieu saint suite à la découverte de vestiges attestant de la Rencontre du Christ avec sa Mère et l'établir comme lieu de pèlerinage.

\section{Attestation de la sainteté du lieu}

Toumayan commence par délimiter le territoire de l'Église arménienne catholique en traçant une frontière avec les zāwiya-s voisines par l'érection d'un mur, à la demande des autorités publiques. La mise en place des fondations constitue un moment fort puisqu'elle va permettre de mettre au jour des vestiges du passé. Situé sur l'itinéraire du Chemin de croix, effectué tous les vendredis par les franciscains, le lieu est connu (selon la tradition) pour abriter le souvenir de la première chute du Christ sous la croix et le souvenir de la Rencontre avec sa Mère qui s'évanouit de douleur. Un archéologue français, Charles ClermontGanneau, en mission pour le compte du Committee of the Palestine Exploration Fund, est mis à contribution pour explorer le site du hammam. Les fouilles sont financées par le père Augustin Albouy, directeur de la revue La Terre sainte, qui soutient fortement toutes les initiatives de développement du catholicisme oriental ${ }^{15}$. Les résultats sont peu probants : un pavement de mosaïque byzantine à motifs géométriques, une citerne et divers objets (Clermont-Ganneau, 1899).

15. Les éléments biographiques concernant ce personnage, qui semble avoir joué un rôle majeur dans la construction du lieu, sont minces. L'ayant connu lors de ses visites à Jérusalem, Toumayan avait sollicité son aide dans une lettre datée du 18 décembre 1880 (La T.S., 15 janvier 1881 : 592). Albouy avait répondu favorablement à son appel en créant l'CEuvre de NotreDame du Spasme pour recueillir les dons des lecteurs. Par ailleurs, ayant été chanoine de Smyrne, il a sans doute eu l'occasion de connaître la population arménienne de la ville. 
En 1881, Toumayan poursuit lui-même l'excavation, cette fois dans la crypte, qui s'avère plus concluante. Il découvre, à sept mètres de profondeur, un panneau de mosaïque byzantine orné d'une paire de sandales; il y décèle les empreintes des pas de Marie, preuve irréfutable à ses yeux de la localisation de la $\mathrm{IV}^{\mathrm{e}}$ station. Des inscriptions grecques et latines viennent renforcer cette thèse bien que seules trois lettres latines aient été déchiffrées : «s-m-o ». Il n'en faut pas plus pour décréter qu'il s'agit du mot "spasimo » et de l'église Notre-Dame du Spasme signalée par plusieurs pèlerins avant sa destruction à l'époque ottomane (Sarkissian, op. cit.) Avec cette découverte, Toumayan inscrit son œuvre dans la continuité de celle des premiers moines, parmi lesquels plusieurs Arméniens, ayant localisé les lieux saints.

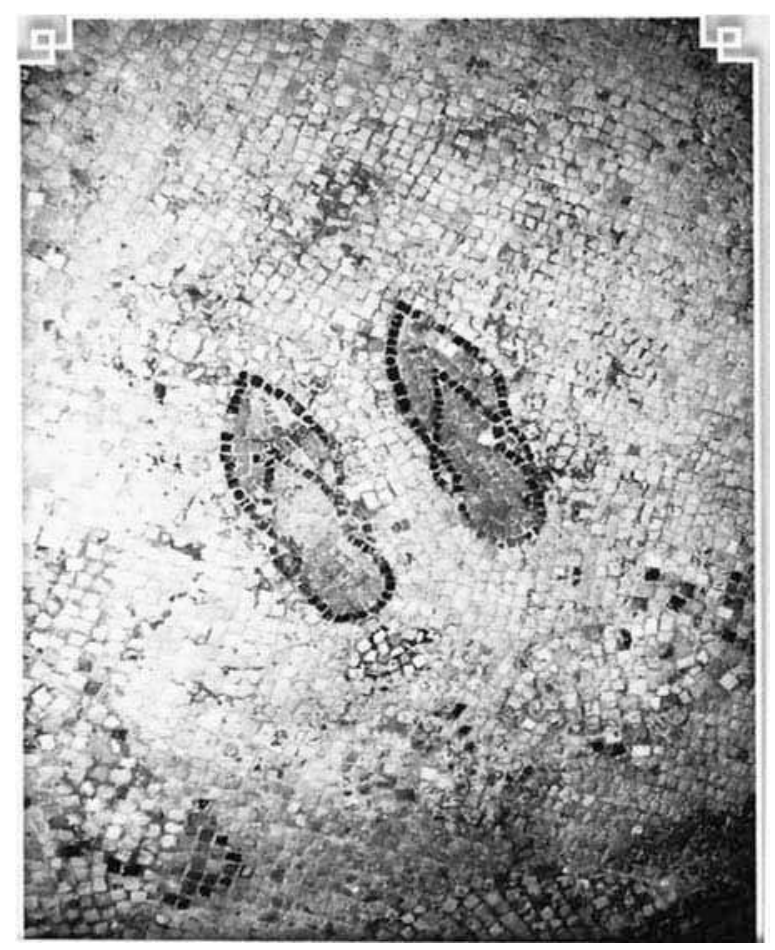

La mosaïque des deux sandales. Crypte de la IV station (Photo S. Andézian)

Albouy publie des notes du père Aigients, missionnaire en Syrie, retraçant l'histoire de l'église ${ }^{16}$. L'auteur confirme la "tradition constante à Jérusalem » selon laquelle la Vierge tombe évanouie de douleur à la vue de son Fils sortant du prétoire, pliant sous le poids de la croix, la tête couronnée d'épines et le visage recouvert de crachats. Les récits des pèlerins localisent ces faits de manière

16. La T.S., ibid.: 652 . 
plus ou moins approximative autour du prétoire, mais tous évoquent l'existence d'un oratoire, d'une chapelle ou d'une église, debout ou en ruine. Aigients recense une dizaine d'ouvrages publiés entre 1306 et 1698 qui permettent de suivre l'évolution de l'édifice. Appelée Sainte-Marie du Spasme, église du Spasme, église de la Pamoyson de la Vierge Marie, sa construction est attribuée, comme tous les monuments chrétiens de Jérusalem, à sainte Hélène, mère de l'empereur Constantin et datée du $\mathrm{IV}^{\mathrm{e}}$ siècle. Après la conquête ottomane, les voyageurs signalent la destruction de l'église ou de son ensevelissement sous de nouveaux bâtiments. Mais, soulignent-ils, tout ce qui s'élève à la place de l'église s'effondre : maisons, bains, écuries, signe du refus de la Vierge de laisser profaner le lieu.

Des légendes se développent autour du hammam, justifiant la nécessité d'y édifier une église. Selon des témoignages recueillis par Toumayan, des fantômes blancs, à l'apparence de personnes âgées enveloppées dans des draps, effrayaient les clients qui s'échappaient tout nus en courant. La fréquentation se réduit et les bains sont abandonnés et tombent en ruine, si bien que le propriétaire vend le terrain. "J'ai entendu ces histoires de mon père mais aussi de beaucoup de personnes âgées, musulmanes et chrétiennes... Au début, je n'y croyais pas ; j'ai commencé à y croire lorsque, au cours du travail de construction, j'ai vu les mille et un obstacles insurmontables se dissiper peu à peu de manière inespérée. Ma foi s'est affermie lorsque j'ai appris que par un miracle de la Mère douloureuse, deux malades incurables avaient été guéris en France. Et j'ai reçu un don de 5500 francs. »(Sarkissian, op. cit. : 514). La légitimité du site choisi par l'Église arménienne catholique à Jérusalem pour établir sa présence en Terre sainte ne fait plus de doute.

\section{(Re)création d'un lieu de pèlerinage}

Aux éléments constitutifs d'un lieu de pèlerinage (tradition, relique, miracles), Toumayan ajoute une statue représentant la scène de la rencontre, commandée en France par l'intermédiaire du père Albouy. Celui-ci annonce à ses lecteurs l'exécution d'un "beau groupe de grandeur héroïque » par la maison Raffl à Paris, spécialiste de sculptures religieuses. Il met en vente un modèle réduit pour quarante francs ou contre un don de cent francs à l'CEuvre ${ }^{17}$. La revue se fait également l'écho de "miracles » produits en France grâce à Notre-Dame du Spasme, des guérisons d'enfants notamment. Une requête d'indulgence est soumise au pape afin de légitimer le site de pèlerinage. Les pèlerins qui effectuent le Chemin de croix se recueillent désormais dans la crypte, où la IV $^{\mathrm{e}}$ station est devenue un lieu d'expérience directe de cette scène de la Passion, tangible et palpable.

17. A. Albouy, "Nouvelles du Spasme ", La T.S., 15 octobre $1881: 795$. 


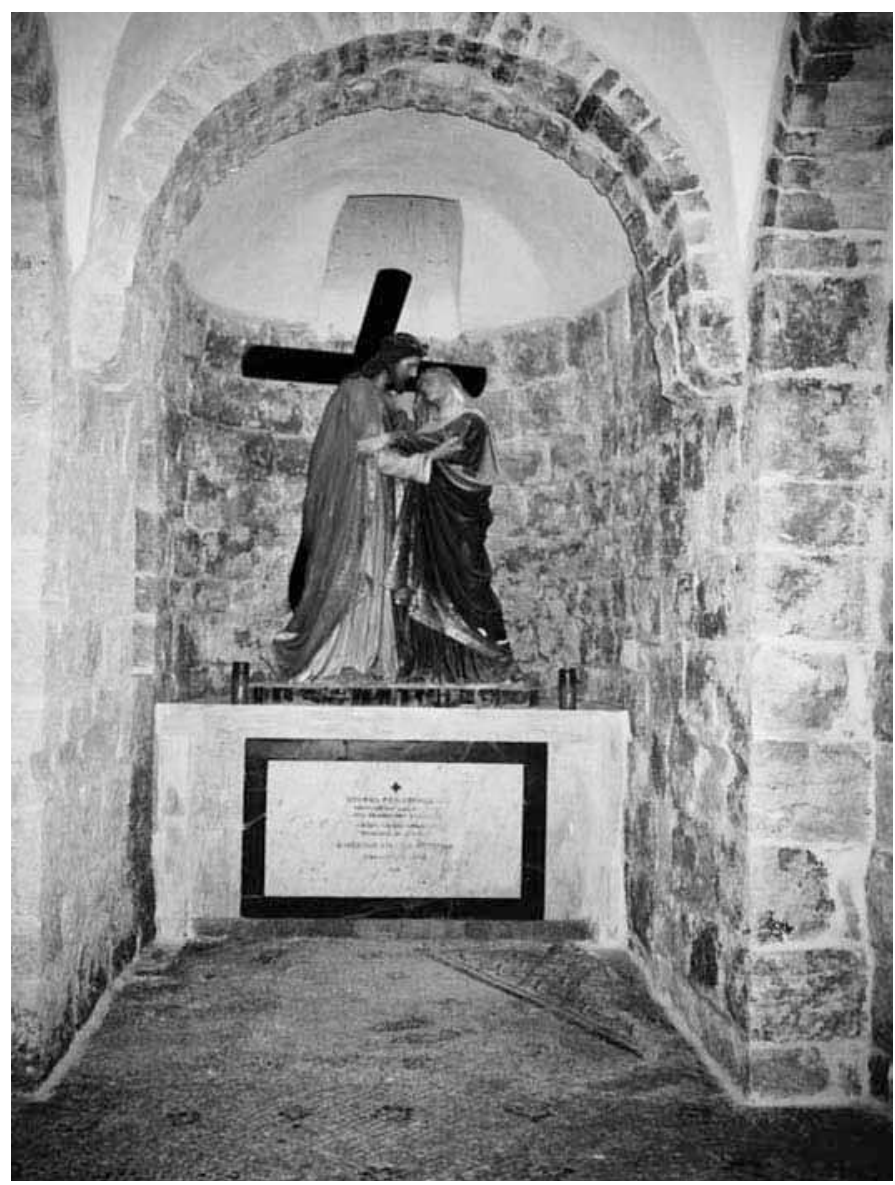

Statue de la Rencontre de la Mère et du Fils. Crypte de la IV station (Photo S. Andézian)

Une autre statue viendra s'y ajouter plus tard, celle de la Vierge de Pontmain, apportée, en 1901, par le père Daireaux, du diocèse de Bayeux et Lisieux, pour la faire bénir et la ramener en France. Celle-ci a un vif succès auprès des fidèles arméniens, qui veulent la garder. Le prêtre leur en fait cadeau, à condition de «bien étudier, pour une propagande mondiale, la raison du culte spécial [qu'on] doit avoir pour l'apparition de Pontmain ${ }^{18}$.

Cependant, la compétition est grande entre Églises catholiques orientales qui cherchent à attirer les pèlerins étrangers. Daireaux mentionne que le père Mallouk, vicaire grec catholique, s'empare de la statue au cours de la procession à travers les rues de la vieille ville et la dépose dans la VI ${ }^{\mathrm{e}}$ station, propriété de son Église.

18. Lettre du 29 mars 1933 du père Daireaux à l'évêque Hovsep Moumdjian, vicaire de 1921 à 1929, rencontré à Rome (arch. vic.) 
Dix ans auparavant, il avait demandé l'aide du père Albouy pour y construire une église à l'instar des Arméniens catholiques ${ }^{19}$.

Dès le début, Toumayan avait sollicité la générosité des catholiques pour financer les travaux : rénovation de la chapelle de la III $^{\mathrm{e}}$ station, déblaiement de la crypte et construction de la chapelle de la IV e station. Le catholicos-patriarche Stepan Azarian l'avait autorisé à ouvrir une souscription générale ${ }^{20}$. L'appel s'adressait en particulier aux catholiques de France, "Fille aînée de l'Église ». La revue La Terre Sainte rendait régulièrement compte de l'état d'avancement des travaux en publiant la liste des noms des bienfaiteurs avec les montants des dons.

La création d'un lieu de pèlerinage sur le site devient d'autant plus urgente que les pèlerinages catholiques connaissent un net développement dans un espace dominé par les pèlerinages orthodoxes (Nicault, 2008). Les pèlerins catholiques, en quête de contact direct avec les lieux sanctifiés par le Christ, mais aussi fascinés par l'Orient, affluent en Terre sainte au cours de cette période. Le pape Léon XIII confie aux assomptionnistes, organisateurs des pèlerinages mariaux à Lourdes et à La Salette dans les années 1870, l'encadrement des pèlerins à partir de 1882 et accorde grâces et indulgences. Deux fois par an (Noël et Pâques), ceux-ci conduisent des pèlerinages de pénitence avec la participation de religieux et de laïcs, en provenance de France principalement. La crypte du Spasme est un des lieux privilégiés de l'exaltation religieuse ${ }^{21}$. Le presbytère, achevé en 1885, accueille les pèlerins en attendant la construction d'un hospice. Comme les pèlerinages des établissements assomptionnistes et de celui des Pères Blancs, celui de la IV ${ }^{\mathrm{e}}$ station s'inscrit dans le prolongement du culte marial en France. Mais le nombre de pèlerins catholiques restera en deçà de celui des orthodoxes (principalement russes) ${ }^{22}$. Comment le lieu s'installe-t-il dans la durée ? Par la légalisation de son existence par les autorités publiques et par son inscription dans la tradition catholique de la ville.

\section{Ancrage en Terre sainte}

Le firman autorisant la construction arrivera en 1887 seulement alors que les travaux sont très avancés. Il est obtenu grâce à l'intervention du patriarche Azarian, connu pour être un fin diplomate et pour avoir servi d'émissaire du

19. La T.S., $1^{\mathrm{er}}$ novembre $1891: 322$.

20. Bulle du 25 mars 1882 portant le sceau du patriarcat arménien catholique de Constantinople et approuvée, le 16 avril 1882, par le patriarche latin de Jérusalem Vincent Bracco (arch. vic.)

21. Le lieu est mentionné assez rapidement dans les guides de pèlerinage.

22. Comme me l'a fait remarquer D. Trimbur, l'objectif de réunir un millier de pèlerins sera réalisé, pour la première fois, seulement en 1882 . La baisse conduira à une sorte de déperdition de sens pour la présence assomptionniste à Jérusalem. Le chiffre de dix mille pèlerins sera atteint à l'occasion du Congrès eucharistique de Jérusalem, en 1893. 
sultan auprès du pape (Hajjar, 1979). Il figure dans le registre ottoman des Églises Kilise Defterleri (Abu Husayn, Saadawi, 1998 : 41-42) qui réunit les décisions des sultans relatives à l'édification d'institutions chrétiennes ottomanes et non ottomanes de l'Empire, entre 1869 et 1922. Selon les sentences (madbata-s) du tribunal, le terrain est composé de deux lots, l'un privé, l'autre waqf. Même s'il touche aux deux zāwiya-s et qu'il est éloigné de soixante coudées seulement du Haram al-Sharif, l'interdiction de construire peut être levée si une rue les sépare. Destinée aux pèlerins provenant d'Istanbul et d'ailleurs, l'église sera "à égalité " (de statut) avec les établissements chrétiens voisins. La première madbata prescrit les dimensions de l'édifice ainsi que le nombre de pièces, la seconde détermine les taxes à payer pour la partie du terrain waqf. Sur la base de ces deux sentences issues du ministère de l'Intérieur et du Conseil d'État, le sultan ordonne au gouverneur de Jérusalem de ne pas interdire la construction de l'église à condition que les dimensions soient respectées et que le patriarcat arménien catholique subvienne aux frais, de ne pas soutirer d'argent à cette communauté et de ne pas lui causer d'ennuis. Grâce au firman, l'Église arménienne catholique acquiert une existence légale à Jérusalem et devient propriétaire et gardienne d'un lieu saint dans un quartier prestigieux.

L'attribution d'un permis de construire une église arménienne catholique à Jérusalem illustre la politique de dérogation aux conditions définies pour l'obtention d'une telle faveur : hors des quartiers musulmans, distant des lieux de culte musulmans, loin des citadelles du sultan. Des aménagements sont possibles en cette fin de XIX ${ }^{\mathrm{e}}$ siècle où le pouvoir ottoman cherche à satisfaire les puissances occidentales (Trimbur, op. cit.)

Le vicaire entreprend l'édification d'une église au-dessus de la crypte conformément au firman ${ }^{23}$. Il continue d'exhorter les catholiques d'Occident et « surtout ceux de la France très chrétienne, à qui revient le glorieux droit de protéger les Lieux Saints ", à faire des dons : "Faudra-t-il que ce firman, accordé par le Sultan, reste sans effet? Quelle responsabilité pour les chrétiens! Le chef de l'Islam aplanit toutes les difficultés et délivre aux mains du patriarche un acte solennel, en vertu duquel un sanctuaire catholique pourra être élevé sur le lieu même de la rencontre douloureuse de la divine Mère avec son Fils, et les catholiques se refuseraient à faire quelques sacrifices pour contribuer à la construction de ce même sanctuaire ? " ${ }^{24}$. Il s'adresse en particulier aux mères et met en exergue la date du firman, un 18 septembre, fête de Notre-Dame des Douleurs ${ }^{25}$

23. C'est l'assomptionniste Étienne Boubet, architecte, qui fera les plans de l'église, en 1891. Venu en pèlerinage à Jérusalem, en 1890, il y était resté et avait conçu les plans de plusieurs autres églises catholiques, telles que l'église Notre-Dame de France et l'église SaintPierre en Gallicante des assomptionnistes.

24. La T.S., 10 octobre $1887: 1007$.

25. Cette fête est fixée au 15 septembre, mais l'Église arménienne la célèbre le dimanche précédant ou suivant cette date (au plus près) comme toutes les principales fêtes. 
(nom choisi pour l'église), "signe du ciel ». Il leur promet la protection de la Vierge et, à partir de mille francs, de graver leur nom sur une plaque de marbre. Des bienfaiteurs s'engagent à faire une offrande annuelle en échange d'une chambre à vie au vicariat ou de messes perpétuelles.

La construction est retardée faute de moyens suffisants. Elle sera achevée en 1905 seulement.

\section{Le lieu dans la tradition catholique de Jérusalem}

Une étude minutieuse de l'archéologue et dominicain Louis-Hugues Vincent (1922) du point de vue architectural, archéologique et historique du site contribue à l'inscrire dans la tradition catholique de la ville, tout en écartant les éléments mythiques.

Vincent fait remonter au XII" siècle la localisation d'une petite église "SainteMarie de Pamoyson dédiée au spasme (tramorticio) de la Vierge »(p. 616), en s'appuyant sur l'origine franque de l'appellation "Pamoyson » et sur l'architecture de la chapelle, carrée, de plan cruciforme et à coupole centrale, qu'il apparente au style des édifices chrétiens de Jérusalem de l'époque. Les éléments archéologiques s'avèrent plutôt minces. Le pavement de mosaïque, découvert par Clermont-Ganneau, ne fournit aucune indication de date ni de destination. Pas plus de données pour déterminer la nature ni la date de réalisation de la mosaïque des deux sandales "silhouettées en noir sur le fond blanc" (ibid.: 600). Leur forme, identique à celles observées sur des mosaïques thermales, ne fournit aucun indice. En revanche, le contexte archéologique évoque la mosaïque funéraire de Saint-Pierre en Gallicante, les deux panneaux ayant pu être réalisés aux $\mathrm{V}^{\mathrm{e}}-\mathrm{VI}^{\mathrm{e}}$ siècles, éléments de décoration des deux églises, identifiées l'une et l'autre sur la mosaïque de Madaba ${ }^{26}$. La construction d'une chapelle au XIII siècle avait sans doute pour objectif, conclut-il, de préserver et de consacrer les vestiges de la mosaïque des deux sandales, "peut-être accidentellement retrouvée ».

Devant l'indigence des preuves, l'auteur scrute les détails de la mosaïque pour en saisir la signification. Il voit dans l'orientement est-ouest des sandales une attestation de son symbolisme religieux, point où les pèlerins se plaçaient pour accomplir les rites. La figuration des pieds, des empreintes de pieds ou de sandales représente dans toutes les religions le souvenir de passage sur les lieux.

Vincent inscrit la construction d'une église sur le site dans le processus plus général de localisation des souvenirs de la Passion et de l'invention de la tradition du Chemin de croix. Non évoqué dans les évangiles, ce rituel se met progressivement en place à partir du XIII ${ }^{\mathrm{e}}$ siècle. Il suscite de l'intérêt lorsque les franciscains reproduisent les souffrances du Christ dans le cadre d'un exercice spirituel.

26. Carte en mosaïque du $\mathrm{VI}^{\mathrm{e}}$ siècle découverte dans la ville jordanienne de Madaba, en 1896, sur laquelle figurent les principaux monuments de Jérusalem de l'époque et qui constitue une source de géographie historique très précieuse. 
L'auteur identifie quatre phases dans le processus de constitution du Chemin de croix à Jérusalem : avant le XIII ${ }^{\mathrm{e}}$ siècle, de 1200 à 1350, de 1350 à 1550, du $\mathrm{XIV}^{\mathrm{e}}$ siècle au début du XX $\mathrm{X}^{\mathrm{e}}$ siècle. Au cours de la deuxième phase, les éléments du Chemin de croix actuel existent déjà mais on cherche à déterminer le parcours du Christ entre le prétoire et le calvaire. Se fondant largement sur les travaux de l'auteur, Halbwachs (2008) avait identifié deux traditions contradictoires ${ }^{27}$. La première, dominante, situait le prétoire dans un palais construit par Hérode sur le mont Sion, au sud de la colline occidentale de la ville, la seconde le situait au nord-ouest du Haram al-Sharif, dans la forteresse d'Antonia. Mais comme des chrétiens locaux continuent de visiter le prétoire à l'emplacement de l'Antonia, cette tradition finit par s'imposer aux Occidentaux ${ }^{28}$. Les tentatives se multiplient pour retrouver l'itinéraire " exact». Celui-ci se constitue en dehors de Jérusalem. Certains de ses inventeurs n'ont jamais visité la ville sainte, tel Jean Pascha, originaire de Louvain, qui met au point un itinéraire mystique en synthétisant les données existantes. Le Chemin de croix est fixé vers le milieu du $\mathrm{XV}^{\mathrm{e}}$ siècle. Les localisations prennent le nom de stations, au sens liturgique de l'Église romaine, marquées par des arrêts de prières au cours des processions pour gagner des indulgences ${ }^{29}$. C'est ainsi que l'église de Pamoyson, dont le souvenir se maintient depuis le XIII ${ }^{\mathrm{e}}$ siècle même après sa destruction, deviendra la $\mathrm{IV}^{\mathrm{e}}$ station. Au XIX siècle, l'initiative de la France et du patriarche latin de réinvestir la Via Dolorosa en y construisant des églises fixera définitivement ces stations.

Après la découverte de la mosaïque des sandales, Toumayan avait fait relayer par la revue La Terre Sainte des messages à tonalité affective, cherchant à susciter l'émotion des lecteurs et exaltant l'amour maternel, le devoir filial et la solidarité entre catholiques. Les miracles évoqués relevaient plutôt de signes annonciateurs de bons présages. Le travail de Vincent permettra de "réajuster » la tradition rattachée au site. Cependant, son installation dans la durée semble avoir nécessité l'élaboration d'un sens mystique mais aussi l'attribution d'une mission autre que l'accueil des pèlerins : celle de réaliser le "retour des Églises dissidentes », en particulier celui de l'Église arménienne. L'action la plus significative sera lancée au début des années trente par le vicaire Hagop Guiragossian (1930-1949), connu comme le rénovateur du lieu et de sa vocation.

27. Halbwachs effectue deux séjours en Terre sainte, du 17 au 19 juin 1927, puis du 29 octobre au $1^{\text {er }}$ novembre 1939. Mais il fait un travail très important de lecture critique des sources françaises, allemandes et britanniques, principalement œuvres d'archéologues. Son ouvrage doit beaucoup à L.-H. Vincent, aussi bien pour les descriptions de sites que pour les analyses.

28. Soulignons que l'Église arménienne apostolique, qui possède le site du prétoire sur le mont Sion, a toujours défendu cette tradition-ci qui fait aujourd'hui la quasi-unanimité parmi les spécialistes.

29. Les stations sont au nombre de quatorze. Cinq d'entre elles ne correspondent pas à un épisode évangélique de la passion (les trois chutes de Jésus, sa rencontre avec sa mère et celle avec Véronique), http://catholique-nanterre.cef.fr/faq/priere_devotions.htm 


\section{Site de pèlerinage, centre communautaire, lieu de réunion des Églises}

Engagé dans le mouvement de diffusion du catholicisme oriental ${ }^{30}$, Guiragossian met en place le Comité Pro-Sanctuario à l'occasion des célébrations des mille cinq cents ans du concile d'Ephèse, en 1931, et des mille neuf cents ans de la Rédemption, en 1933-1934. Il ne s'agit pas d'un simple appel de fonds pour décorer et meubler l'église, mais de l'adoption d'une doctrine rattachée au lieu. L'entreprise est soutenue par Rome, le délégué apostolique, le patriarche latin et le patriarche arménien catholique.

Inspirée de l'Encyclique Lux Veritatis ${ }^{31}$, cette doctrine est présentée dans un texte en italien de dix-huit pages, non daté et non signé mais indiquant son émanation du vicariat ${ }^{32}$. Placé dans l'histoire longue du christianisme, "le modeste sanctuaire ", dont la description est empruntée à l'ouvrage de Vincent (op. cit.), est présenté comme un lieu synthétisant les deux célébrations solennelles et réunissant la dévotion à Marie et la Rédemption ${ }^{33}$. Mais ce lieu a un mérite supplémentaire, souligne l'auteur, celui d'être en pratique un centre d'activité pour le retour au bercail de tous les dissidents, spécialement des Arméniens avec lesquels il est plus directement en relation. Le sanctuaire représente non seulement deux idéaux de la piété chrétienne, mais également "l'idéal qui fut le grand amour du Christ et de Marie, la réunion de toutes les âmes dans une seule bergerie dirigée par un seul pasteur» (p. 7). Et la douleur de la Vierge à la vue de son Fils portant la Croix devient celle de la Vierge à la vue de ses enfants séparés : "Elle qui est tant aimée et tellement honorée par les Orientaux dissidents ne permet pas que ceux-ci soient malheureusement détournés et qu'ils s'éloignent toujours plus de l'Unité de l'Église et de son Fils. Que le Ciel fasse qu'arrive ce jour heureux où la Vierge Marie pourra voir le retour des enfants de nos séparés, pour la vénérer ensemble avec nous, avec une seule âme et une seule foi » (p. 12).

Puis l'article insiste sur le caractère providentiel de l'attribution à l'Église arménienne catholique de la propriété "d'où a surgi le sanctuaire ", "lui confiant également la mission de contribuer de toutes ses forces à la conservation de la foi chez les Arméniens catholiques résidant en Palestine et à l'apostolat pour le retour à l'unité de l'Église de la plupart des dissidents et particulièrement des

30. En témoignent les nombreux textes de réflexion à l'état de brouillons dans les archives du vicariat.

31. Encyclique de Pie XI pour le $\mathrm{XV}^{\mathrm{e}}$ centenaire du concile d'Éphèse. Voir le texte en français : http://www.ddata.over-blog.com

32. "Sanctuario dello "Spasimo" della Beata Vergine Maria sulla Via Dolorosa a Gerusalemme» (arch. vic.)

33. Au concile d'Éphèse (431), Nestorius et Cyrille d'Alexandrie s'étaient affrontés au sujet de l'union des deux natures divine et humaine du Christ ainsi que du titre Theotokos attribué à la Vierge. 
Arméniens schismatiques qui se trouvent en Terre sainte » (pp. 12-13). Le choix divin n'est pas anodin, poursuit l'auteur, puisque l'Église arménienne catholique est l'Église martyre par excellence en raison de sa fidélité inébranlable à Rome. En outre, elle a une longue expérience des dissidents en son sein et est à même de comprendre l'âme des Orientaux.

À cette fin est envisagée la fondation d'une confrérie : Notre-Dame des Douleurs pour l'Union des dissidents. L'admission dans la confrérie se fait par simple inscription. Il est recommandé de réciter quotidiennement trois Ave Maria et des prières prescrites, de faire de bonnes actions, des sacrifices et des dons (argent, objets liturgiques, mobilier...) Les noms des bienfaiteurs qui offrent au moins une livre sterling seront gravés sur une plaque de marbre apposée sur une paroi de la crypte. Les plus généreux d'entre eux jouiront de la mémoire perpétuelle, avec leur nom et prénom gravés sur une table de marbre. Le pape accorde des indulgences en attendant l'autel principal auquel il confèrera l'indulgence plénière.

Dans les faits, le travail du vicaire se limitera à tenter de développer le catholicisme parmi les Arméniens. L'arrivée de rescapés du génocide, dans les années vingt, dont plusieurs de rite arménien catholique, avait nécessité la création, parallèlement au centre de pèlerinage, d'un centre communautaire pour répondre aux besoins matériels, culturels et spirituels de ceux des réfugiés que le patriarcat apostolique ne pouvait accueillir. Son prédécesseur, Moumdjian, avait cherché à identifier ceux des réfugiés du monastère apostolique de rite catholique ou souhaitant l'adopter. Par ailleurs, avec l'accord du patriarche latin, il invitait les Arméniens de rite latin à célébrer baptêmes, mariages et funérailles selon le rite arménien catholique; leurs noms figurent ainsi dans les registres de la paroisse. Son projet de construire une école n'aboutira pas durant son mandat. En revanche, une association culturelle verra le jour grâce à la participation active de plusieurs intellectuels arméniens catholiques qui diffuseront la langue et la culture arméniennes parmi les réfugiés, majoritairement turcophones (arch. vic.)

La communauté arménienne catholique prend véritablement forme avec le père Guiragossian. Elle bénéficie à la fois de la politique communautariste du gouvernement britannique et de l'impulsion des associations politiques et culturelles arméniennes à reconstruire les infrastructures nationales dans les pays d'adoption. Le rôle des Églises sera particulièrement important dans la mise en place du nouveau cadre de vie des réfugiés. En Palestine, comme ailleurs dans les pays arabes héritiers du système ottoman, les chefs religieux ont le statut de chefs communautaires. Et c'est à ce titre que le vicaire de Jérusalem devient le porte-parole des Arméniens catholiques auprès de l'administration britannique et intervient dans la gestion de tous les aspects de leur vie : état civil, législation, emploi, aide sociale, éducation, vie associative... Sa correspondance très fournie avec ces différentes instances témoigne de son action. Il organise la paroisse qui, jusque-là, avait fonctionné de manière informelle. Au sein du vicariat même, il entreprend la modernisation des bâtiments et l'installation de l'électricité et de 
l'eau courante. Il déploie beaucoup d'énergie pour l'ouverture d'une école, qui sera encadrée d'abord par des moines mékhitaristes de Venise, puis par les sœurs de la congrégation arménienne de l'Immaculée conception. Une imprimerie permet de publier le journal Yerusaghem (Jérusalem) qui sera également diffusé parmi la diaspora. Comme le font toutes les autres Églises de Terre sainte, il crée des associations sportives et culturelles, un groupe scout, une fanfare, initiatives favorisées par le gouvernement britannique : leur participation à toutes les manifestations officielles tend à donner une plus grande visibilité à la communauté. Une compétition se crée ainsi entre les deux Églises arméniennes pour gagner des fidèles et assurer leur éducation générale et religieuse mais aussi pour préserver leur identité nationale ( $\mathrm{arch}$. vic.)

Toutefois les conversions au rite arménien catholique seront peu nombreuses. Le patriarcat arménien apostolique ainsi que les associations politiques et culturelles s'opposent à ce qu'ils désignent comme mouvement de "latinisation ", terme fortement connoté dans l'histoire de l'Église arménienne, à un moment de grande mobilisation pour reconstruire la nation. Des familles qui étaient passées au rite catholique retournent au rite apostolique. De son côté, un franciscain arménien interdit aux parents de retirer les enfants de l'école franciscaine pour les envoyer à l'école arménienne catholique, sous peine de leur enlever l'aide matérielle et/ou le logement octroyés par la Custodie ${ }^{34}$.

L'analyse du positionnement de l'Église arménienne catholique dans le champ du christianisme à Jérusalem est révélatrice de sa stratégie de construction d'une identité singulière. Si elle adopte la doctrine romaine, elle conserve la liturgie et la langue arméniennes et recouvre l'église de symboles nationaux. Et tout en cherchant à attirer les pèlerins occidentaux, les vicaires veilleront à préserver l'identité arménienne du lieu ${ }^{35}$ : Toumayan sera même soupçonné d'avoir voulu se l'approprier. Cette figure est emblématique de la complexité des rapports que les catholiques autochtones entretiennent avec la Terre sainte, d'une part, avec la hiérarchie romaine et celle de leur Église nationale, d'autre part.

34. L'espace de cet article ne permet pas de développer plus avant cette partie, qui nécessiterait un article en soi.

35. Au fil du temps, les symboles arméniens seront plus manifestes dans le cadre bâti. L'autel central de l'église, en forme de croix latine, sera protégé par une structure caractéristique de l'architecture sacrée arménienne : une coupole de pierre sur tambour polygonal soutenu par quatre pilastres massifs, surmontée d'une coiffe conique portant une croix. Une inscription en arménien indique que l'autel, consacré en 1972, est dédié aux martyrs du génocide du 24 avril 1915. Une autre pièce d'architecture arménienne, la croix de pierre ou khatchkar, stèle sur laquelle est ciselée une croix décorative devenue l'emblème du souvenir du génocide, s'élève sur le parvis depuis 2005. En 1965, lorsque les Arméniens de la diaspora commémorent le cinquantenaire du génocide, devenue un rituel annuel depuis, une cérémonie du souvenir est organisée par le vicaire arménien catholique à laquelle participe l'ensemble des Arméniens de Terre sainte. 


\section{Le père Hovaguim Toumayan, figure emblématique}

Dans les années trente, le lieu était connu dans le quartier comme la maison du Père Hovaguim (Toumayan), en arabe dār abūna Wakim (Stephan, op. cit.) La mémoire de l'Église a également associé le nom de ce vicaire à la fondation du lieu, occultant celui de Tavitian qui avait acheté le terrain et construit la chapelle de la troisième station. De tous les vicaires, Toumayan est le seul enfant du pays, né à Jérusalem (1848), dans une famille arménienne qui se réclame de la lignée du roi Héthoum ${ }^{36}$. Il fait partie des Arméniens de la ville qui revendiquent leur autochtonie par rapport à ceux arrivés après le génocide, désignés par le terme arabe zuwwār , visiteurs ou pèlerins. Il en connaît les langues et les mœurs, de même qu'il dispose d'un réseau de relations étendu dans cette ville cosmopolite de la seconde moitié du XIX ${ }^{\mathrm{e}}$ siècle. Il compte parmi ses relations des membres des grandes familles musulmanes exerçant de hautes fonctions au sein du gouvernement, la famille Khalidi par exemple. De par son histoire personnelle, il est ancré à la fois dans la société orientale et les milieux des missionnaires occidentaux. Il peut ainsi mobiliser réseaux locaux et réseaux internationaux en cas de nécessité.

Cette posture est en même temps source de tensions et a pu entraver la réalisation de ses projets. De rite arménien apostolique, sa famille adopte le rite latin à la suite d'un conflit avec un prêtre du patriarcat arménien apostolique. Expulsée du logement qu'elle occupait dans le quartier arménien, elle trouve refuge chez les franciscains. Le jeune Kévork (son nom de baptême), qui est scolarisé chez ces derniers, exprime dès l'âge de dix ans le désir d'entrer dans l'ordre. Sa demande est rejetée au motif que la congrégation n'admet pas de novices originaires de Jérusalem. En réalité elle n'accepte pas les novices indigènes, qui sont invités à rejoindre la branche catholique de leur Église nationale. C'est ainsi que le patriarche latin l'adresse au prêtre arménien catholique qui vient de s'installer dans la ville. Ce dernier le conduit au séminaire de Bzommar où il est ordonné prêtre en pleine crise de l'Église. Il en est tellement affecté qu'il demande son retour à Jérusalem, en 1868. Mais, se sentant rejeté, il repart au Liban au bout de quatre mois. Il s'installera définitivement à Jérusalem en 1872. En attendant la construction du presbytère, il loge, comme son prédécesseur, au patriarcat latin. Même s'il porte le titre de vicaire patriarcal, il se retrouve seul face aux difficultés liées à l'établissement du centre de pèlerinage et de l'église, ses supérieurs hiérarchiques étant trop accaparés par leurs problèmes internes ${ }^{37}$. Il consacre sa vie à la construction de l'église, entreprise qui dure plus de vingt ans, avant d'être rappelé au Liban où il est nommé supérieur du séminaire.

36. Le roi Héthoum II de Cilicie conquiert Jérusalem de 1300 à 1303. Dans les faits, beaucoup d'Arméniens se sont installés à Jérusalem à l'époque des croisades.

37. Document autobiographique inédit en arménien ancien (arch. vic.) 
Ses adversaires lui avaient reproché de s'être approprié le lieu et d'avoir accumulé les dettes, alors que lui-même soutient avoir utilisé ses deniers personnels pour financer les travaux. En 1927, fatigué et malade, il demande à vivre ses dernières années dans sa ville natale. Lorsqu'il meurt, le 26 janvier 1929, plusieurs notices nécrologiques, émanant de différentes instances de l'Église catholique ainsi que des établissements religieux locaux, lui rendent hommage pour son œuvre ${ }^{38}$. Sa volonté d'être inhumé dans la crypte en échange de sa renonciation à tout droit au profit de ses héritiers est respectée. Et son nom sera inscrit sur une plaque recouvrant sa tombe, creusée à un mètre de la mosaïque des sandales et à l'ombre de la statue représentant la Rencontre de Jésus avec sa Mère. Au-dessus, une niche vitrée abrite "ses propres reliques ": anneau d'abbé mitré, croix pectorales, médailles honorifiques, évangile, missel.

La présence de ses reliques au voisinage immédiat de celles de la Vierge contribue au marquage de cette portion de territoire sacré par les Arméniens de Jérusalem. Propriétaire légale du lieu saint, l’Église arménienne catholique en devient également la propriétaire légitime. Mais le site constitue un lieu de mémoire pour les Arméniens de tous rites. Ainsi, lors de l'inauguration de la crypte, en 2005, les Arméniens de Jérusalem, en particulier les « autochtones ", découvriront avec fierté ce compatriote, voire parent, plusieurs d'entre eux portant le même patronyme. Et c'est moins en tant que fondateur de l'Église arménienne catholique de Jérusalem qu'ils le revendiquent comme un ancêtre, puisque la plupart d'entre eux appartiennent à l'Église apostolique, que comme une figure éponyme ayant par son œuvre "sanctifié » leur lignée.

\section{Conclusion}

La fondation d'un centre de pèlerinage sur les sites des $\mathrm{III}^{\mathrm{e}}$ et $\mathrm{IV}^{\mathrm{e}}$ stations de la croix suit un modèle classique de fondation de lieux de culte : invention d'une tradition par le recours à des données archéologiques, textuelles et orales ; découverte de reliques ; construction matérielle ; élaboration d'un sens mystique. Son installation dans la durée a nécessité la mise en place d'un rituel et d'une vocation spécifiques. Mais les contextes politique et religieux ont grandement favorisé la réussite de l'entreprise malgré les difficultés qu'ils pouvaient paradoxalement faire naître. Ainsi, l'arrivée des rescapés du génocide a contraint l'Église à créer les infrastructures nécessaires à l'encadrement d'une communauté, ce qui a donné plus d'assise au vicariat en tant que gardien d'un lieu saint. La possession d'un lieu saint à Jérusalem est fortement subordonnée à la présence d'une communauté de fidèles visibles dans l'espace public lors des manifestations solennelles.

38. En particulier, les religieuses du monastère Hortus Conclusus d'Artas, près de Bethléem, qu'il avait aidées pour l'achat du terrain; les sœurs de Sion, ses voisines, à qui il rendait de nombreux services au quotidien. 
Cet exemple illustre le rapport aux lieux saints des habitants de Jérusalem qui se sentent investis de la fonction de gardiens de ces lieux et des traditions qui leur sont rattachées, ainsi que du rôle de représentants locaux des Églises universelles. L'appartenance à une Église locale, voire nationale, n'est pas exclusive de l'appartenance à une Église universelle. Toutefois, la notion de propriété d'un territoire sacré et d'autochtonie demeure très forte, même si on y accueille les pèlerins étrangers. C'est l'un des problèmes récurrents qui se posera avec plus ou moins d'acuité dans les deux stations, à la fois patrimoine de l'Église catholique universelle et domaine privé de l'Église arménienne catholique, mais aussi patrimoine arménien local et patrimoine national.

L'étude du processus de fondation de ce lieu est en même temps pertinente en ce qu'elle révèle les tensions internes à l'Église catholique. En ce qui concerne l'Église arménienne, les tiraillements étaient permanents suite à la politique centralisatrice du Vatican qui a conduit à une série de divisions internes : - fragmentation de la communauté arménienne de Constantinople, par la création d'un millet arménien catholique qui a éloigné des compatriotes, voire des familles, les uns des autres ; - scission au sein de l'Église elle-même par l'établissement d'un second patriarcat, celui de Constantinople, indépendant de celui de Cilicie, puis par le transfert du siège patriarcal dans cette ville, initiatives auxquelles s'étaient opposés une partie du clergé et les laïcs, provoquant un " néo-schisme » avec la création d'une nouvelle Église et l'adhésion de certains à l'Église apostolique ; - éviction des laïcs des instances de l'Église dont la participation était devenue importante à la faveur des tanzimāt. Aussi l'objectif de faire du lieu un centre de retour des schismatiques au catholicisme n'a-t-il pu être atteint. En revanche, le génocide a réuni Arméniens apostoliques et Arméniens catholiques autour du projet de reconstituer la nation.

\author{
Sossie ANDÉZIAN \\ CEIFR (CNRS/EHESS) \\ sandezian@yahoo.fr
}

\title{
Bibliographie
}

Abû Husayn 'Abd al-Rahîm, SA'ADAWî Sâleh, 1998, Al-kanā'is al-'arabiyya fil-sijjil alkanā'isī al-'utmāñ̄ 1869-1922 [Les Églises arabes dans le registre ecclésial ottoman], Amman, Institut royal des études religieuses.

Bouwen Frans, 1992, "Le Cardinal Lavigerie et l'union entre les Églises d'Orient et d'Occident ", Proche-Orient chrétien, 42, pp. 383-405.

Clermont-Ganneau Charles, 1899, Archeological Researches in Palestine During the Years 1873-1874, Published for the Committee of the Palestine Exploration Fund, London, Harrison and Sons, vol. 1.

Djourian Mesrob, 1990, "L’Église catholique arménienne ", in Dédéyan G., (dir.), Les Arméniens. Histoire d'une chrétienté, Toulouse, Privat, pp. 99-109. 
Duvignau Pierre, 1972, Une vie au service de l'Église : S.B. Mgr Joseph Valerga, Jérusalem, Imprimerie du Patriarcat latin.

HaJjar Joseph, 1979, Le Vatican, la France et le catholicisme oriental (1878-1914). Diplomatie et histoire de l'Église, Paris, Beauchesne.

Halbwachs Maurice, [1941] 2008, La topographie légendaire des évangiles en Terre sainte. Étude de mémoire collective, Paris, Presses Universitaires de France.

Mills E., 1931, Census of Palestine, vol. III, Table VII, Part 2.

Mouradian Claire, 1999, "Les chrétiens, un enjeu pour les puissances ", in Nicault C., (dir.), Jérusalem 1850-1948. Des Ottomans aux Anglais : entre coexistence spirituelle et déchirure politique, Paris, Autrement, "coll. Mémoires ", pp. 177-204.

NiCAult Catherine, 2008, Une histoire de Jérusalem 1850/1967, Paris, CNRS Éditions.

Ormanian Malachia (Arch.), [1910] 2000, The Church of Armenia. Montreal, Armenian Holy Apostolic Church, Canadian Diocese (trad. G. Marcar Gregory).

SARKISSIAN Parsegh, 1906, "Hayotz Diramor talgatzmane nevirial noragatzouytz Mayr Tzavakin yegueghetzin y Sourp Yeroussaghem » [Église arménienne Notre-Dame-des Douleurs récemment édifiée dédiée au spasme de la Vierge à Jérusalem la sainte], Pazmaveb, pp. 511-516.

Sroor Musa, 2005, Fondations pieuses en mouvement : de la transformation des statuts de propriété de biens waqf-s à Jérusalem (1858-1917), thèse de doctorat, Université de Provence Aix-Marseille I.

StePHAN Stephan H., 1933, "Three Firmân's granted to the Armenian Catholic Community, Jérusalem », Journal of the Palestine Oriental Society, XIII, pp. 239-246.

Trimbur Dominique, 2000, "Sainte-Anne : lieu de mémoire et lieu de vie français à Jérusalem ", Chrétiens et sociétés XVI ${ }^{e}-\mathrm{XX}^{e}$ siècles, 7, pp. 39-69.

-, 2002, "Vie et mort d'un séminaire syrien-catholique. L'établissement bénédictin de Jérusalem ", in Proche-Orient chrétien, 52/3-4, pp. 303-352.

VINCENT Louis-Hugues, 1922, Jérusalem. Recherches de topographie, d'archéologie et d'histoire, Fasc. III, Paris, Gabalda.

Whooley, John, 2004, "The Armenian-Catholic Church: A Study in History and Ecclesiology ", Heythrop Journal, XLV, pp. 416-434.

\section{Résumé}

L'Église arménienne catholique de Jérusalem a été fondée, au XIXe siècle, à la faveur des réformes ottomanes ainsi que du développement des missions chrétiennes. Implantée dans le quartier musulman, elle est construite sur les ruines d'un hammam, où la tradition catholique situe les $I I I^{e}$ et IV $V^{e}$ stations de la Croix. Lieu de pèlerinage international, c'est également le siège d'un vicariat patriarcal et d'une paroisse pour les Arméniens catholiques locaux. L'analyse du positionnement de cette Église dans le champ du christianisme oriental est révélatrice des tensions résultant de sa stratégie de construction d'une identité singulière, par rapport à l'Église catholique universelle, à l'Église arménienne apostolique et aux autres Églises de Jérusalem. Cet exemple illustre les modalités de réappropriation des lieux saints chrétiens, ainsi que les processus de formation de nouvelles Églises et de nouvelles communautés confessionnelles.

Mots-clés : Église arménienne catholique, Jérusalem, lieux saints, pèlerinage, communauté confessionnelle. 


\section{Abstract}

The Armenian Catholic Church in Jerusalem was founded during the $19^{\text {th }}$ century in the context of Ottoman reforms and the developing of Christian missions. Located in the Muslim quarter, it is built on the ruins of a hammam, where Christian tradition places the III th and the IV $V^{\text {th }}$ stations of the Cross. A site of an international pilgrimage, it is at the same time the see of a patriarchal vicariate and the parish of the local Armenian Catholics. The analysis of the position of this Church in the sphere of Oriental Christianity reveals the tensions resulting from her strategy of constructing a specific identity, distinct from that of the Universal Catholic Church, the Armenian Apostolic Church and the other Churches of Jerusalem. This example illustrates the ways Christian holy sites are reappropriated, as well as the processes of creating new Churches and new confessional communities.

Key words: Armenian Catholic Church, Jerusalem, holy sites, pilgrimage, confessional communities.

\section{Resumen}

En el siglo XIX se funda la Iglesia católica Armenia de Jerusalén bajo el impulso de las reformas otomanas, y siguiendo el desarrollo de las misiones cristianas. Implantada en el barrio musulmán, es construida sobre las ruinas de un hammam, donde la tradición católica sitúa las III ${ }^{a}$ y IV ${ }^{a}$ estaciones de la Cruz. Lugar de peregrinación internacional, es también la sede de un vicariato patriarcal y de una parroquia para los armenios católicos locales. El análisis del posicionamiento de esta Iglesia en el campo del cristianismo oriental es reveladora de las tensiones que resultan de su estrategia de construcción de una identidad singular, en relación con la Iglesia católica universal, la Iglesia Armenia apostólica y las otras Iglesias de Jerusalén. Este ejemplo ilustra las modalidades de reapropiación de los lugares santos cristianos, así como los procesos de formación de las nuevas Iglesias y de las nuevas comunidades confesionales.

Palabras clave: Iglesia Armenica católica, Jerusalém, lugares santos, peregrinación, comunidad confesional. 